\title{
Differential Effects of Sucrose, Fructose, and Glucose on Postprandial Carbohydrate and Lipid Metabolism in Young Japanese Women
}

\author{
Erika Mizutani $^{1,2}$, Sayuki Hashimoto ${ }^{1,3}$, Hiromi Saito ${ }^{1}$, Maiko Kato ${ }^{1}$, Akihiro Yoshida ${ }^{4}$, \\ Michitaka Naito ${ }^{1, *}$ \\ ${ }^{1}$ Division of Nutrition \& Health, School \& Graduate School of Life Studies, Sugiyama Jogakuen University, Nagoya, Japan \\ ${ }^{2}$ Department of Food and Nutrition, College of Nagoya Bunri University, Nagoya, Japan \\ ${ }^{3}$ Department of Food and Nutritional Environment, College of Human Life and Environment, Kinjo Gakuin University, Nagoya, Japan \\ ${ }^{4}$ Department of Clinical Laboratory, Nakatsugawa Municipal General Hospital, Nakatsugawa, Japan
}

Email address:

naito@sugiyama-u.ac.jp (M. Naito)

${ }^{*}$ Corresponding author

\section{To cite this article:}

Erika Mizutani, Sayuki Hashimoto, Hiromi Saito, Maiko Kato, Akihiro Yoshida, Michitaka Naito. Differential Effects of Sucrose, Fructose, and Glucose on Postprandial Carbohydrate and Lipid Metabolism in Young Japanese Women. Journal of Food and Nutrition Sciences.

Vol. 6, No. 1, 2018, pp. 1-11. doi: 10.11648/j.jfns.20180601.11

Received: August 10, 2017; Accepted: September 9, 2017; Published: December 14, 2017

\begin{abstract}
Differential effects of sugars, namely sucrose, fructose, and glucose on postprandial carbohydrate and lipid metabolism were investigated in young women. Young Japanese women with normal body mass (BMI $\geq 18.5-<25 \mathrm{~kg} / \mathrm{m}^{2}$ ) and apolipoprotein $\mathrm{E}$ phenotype $3 / 3$ were enrolled. In the experiment $(\operatorname{Exp}) 1$, subjects $(\mathrm{n}=10)$ ingested one of the beverages containing (1) glucose $(0.5 \mathrm{~g} / \mathrm{kg}$ body mass), (2) fructose $(0.5 \mathrm{~g} / \mathrm{kg})$, (3) sucrose $(0.5 \mathrm{~g} / \mathrm{kg})$, or (4) glucose $(0.25 \mathrm{~g} / \mathrm{kg})+$ fructose $(0.25 \mathrm{~g} / \mathrm{kg})$ in a randomized crossover design. In Exp 2, subjects $(\mathrm{n}=10)$ ingested fat cream (fat content: $0.35 \mathrm{~g} / \mathrm{kg}$ ) alone or along with either beverage (1), (2), or (3). Blood samples were collected at baseline and 0.5, 1, 2, 4, and $6 \mathrm{~h}$ after ingestion and then analyzed. In Exp 1, the ingestion of fructose non-significantly increased postprandial serum triglyceride (TG), hepatic TG-rich lipoprotein-TG, and remnant cholesterol levels. In Exp 2, coingestion of fructose or sucrose with fat delayed postprandial lipidemia compared with the ingestion of fat alone. The extent of postprandial lipid response after coingestion of sucrose with fat was intermediate between that observed after coingestion of fructose with fat and that observed after coingestion of glucose with fat. In conclusion, ingestion of fructose may increase endogenous TG-rich lipoprotein concentration, but the effect of sucrose was weaker. Coingestion of fructose and fat delayed postprandial lipidemia compared with the ingestion of fat alone. The extent of postprandial lipid response by sucrose coingestion with fat was intermediate between fructose and glucose.
\end{abstract}

Keywords: Sucrose, Fructose, Glucose, Triglyceride, Remnant Lipoprotein

\section{Introduction}

Women in reproductive age generally have a lower risk of cardiovascular disease (CVD) than age-matched men. Very-low-density lipoprotein (VLDL) particle number and serum triglyceride (TG) concentration are risk factors for CVD; women typically have lower serum TG concentration than men, which is attributable to lower VLDL-TG concentration [1]. However, serum TG level is an important and independent predictor of coronary heart disease and stroke risk [2]. Moreover, nonfasting TG level is associated with incident cardiovascular events in women, independent of traditional risk factors, whereas the association between fasting TG level and the events has little independent relationship [3]. Furthermore, nonfasting TG level has better predictive ability in women than in men [4]. More importantly, recent Mendelian randomization studies demonstrated that raised TG, remnant cholesterol, or TG-rich lipoproteins is causally associated with cardiovascular disease, independent of reduced high-density lipoprotein-cholesterol (HDL-C) [5, 
6]. The consumption of added sugars is positively associated with indicators that increase CVD risk, including high TG and low HDL-C levels in adolescents [7, 8] and adults [9].

The consumption of sugars has been increasing in Asia. Consumers with the highest intake of sucrose and high-fructose syrup (HFS) are adolescents and young adults in the USA [10], plausibly similar to the situation in Japan. Therefore, we previously studied the effects of fructose and HFS on carbohydrate and lipid metabolism in young Japanese women [11, 12] and found that coingestion of a moderate amount of fructose and fat exacerbated postprandial lipidemia by delaying secretion and clearance of intestinal triglyceride-rich lipoprotein (TRL) [11]. Because fructose is typically consumed as HFS or sucrose, we next examined the effects of ingestion of a fructose-containing beverage with fat and found that coingesting a HFS-containing beverage with fat can delay and exacerbate both exogenous and endogenous lipidemia and that the fructose-to-glucose ratio may determine metabolic disturbance when sugar load is equicaloric [12].

In the present study, we examined the differential effects of fructose, glucose, and sucrose (a compound composed of glucose and fructose in equal amounts) on postprandial carbohydrate and lipid metabolism in young Japanese women.

\section{Methods}

\subsection{Subjects}

Young Japanese women with normal body mass, normal ovarian cycles, and apolipoprotein (apo) E phenotype 3/3 were enrolled. Normal body mass was defined as body mass index (BMI) between $\geq 18.5 \mathrm{~kg} / \mathrm{m}^{2}$ and $<25 \mathrm{~kg} / \mathrm{m}^{2}$. Subjects were nonsmokers, not suffering from any apparent acute or chronic illness, and not taking any medications or dietary supplements. This study was approved by the Institutional Review Board of Sugiyama Jogakuen University School of Life Studies in accord with Helsinki Declaration (No. 2013-3), and each subject provided a written informed consent for participation.

\subsection{Anthropometric and Body Composition Measurement}

Body mass and height were measured using standard methods. Waist circumference was assessed as the abdominal girth at the level of the umbilicus, whereas the hip was measured at the level of the greater trochanters. The waist-to-hip $(\mathrm{W} / \mathrm{H})$ ratio was calculated. Body composition including visceral fat area (VFA) was analyzed by an eight-polar bioelectrical impedance method, using InBody720 (BioSpace, Tokyo, Japan).

\subsection{Sucrose, Fructose, Glucose, and Fat Load Tests}

In Exp 1, subjects $(\mathrm{n}=10)$ ingested one of the beverages containing (1) glucose $(G),(2)$ fructose $(F),(3)$ sucrose $(S)$, or (4) glucose + fructose $(\mathrm{GF})$. In Exp 2, subjects $(\mathrm{n}=10)$ ingested fat cream (OFTT cream ${ }^{\mathrm{TM}}$, Jomo Co., Takasaki, Japan; $1 \mathrm{~g} / \mathrm{kg}$ containing $0.35 \mathrm{~g} / \mathrm{kg}$ of fat) alone (Ft) or along with either beverage (1), (2), or (3) (GFt, FFt, or SFt, respectively). Both experiments used a randomized crossover design. The beverages were prepared at following concentrations: $\mathrm{G}, 0.5 \mathrm{~g} / \mathrm{kg}$ body mass of glucose (Natural Kitchen, Nagoya, Japan); F, $0.5 \mathrm{~g} / \mathrm{kg}$ of fructose (Nisshin Seito, Tokyo, Japan); S, $0.5 \mathrm{~g} / \mathrm{kg}$ of sucrose (Nisshin Seito); GF, $0.25 \mathrm{~g} / \mathrm{kg}$ of glucose $+0.25 \mathrm{~g} / \mathrm{kg}$ of fructose. All beverages were prepared at the same concentration of $10 \%$ $(\mathrm{w} / \mathrm{v})$. OFTT cream $^{\mathrm{TM}}$ was used as described previously [13].

\subsection{Experimental Design}

Subjects ingested one of the test beverages after a 12-h overnight fast (Figure 1). Subjects abstained from caffeine and alcohol intake on the day before the trial. Venous blood samples were taken before $(0 \mathrm{~h})$ and $0.5,1,2,4$, and $6 \mathrm{~h}$ after ingestion. During the test, the subjects avoided exercise and eating, but had free access to water after $1 \mathrm{~h}$ of ingestion. All blood samples were obtained in the supine position. There was an interval of 4 weeks between the test days to minimize the confounding effects of menstrual status on lipid metabolism.

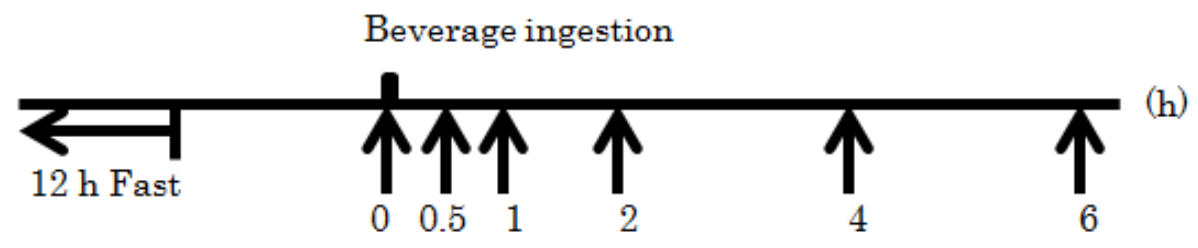

Upward arrows $(\uparrow)$ indicate the time-points of blood sampling.

Figure 1. Protocol of the trials.

\subsection{Biochemical Analysis}

Serum samples were immediately refrigerated $\left(4^{\circ} \mathrm{C}\right)$ or frozen $\left(-80^{\circ} \mathrm{C}\right)$ until analysis. Fructose was measured enzymatically (BioAssay Systems, CA, USA). Glucose was measured by the mutarotase-glucose oxidase method (Wako, Osaka, Japan). Insulin was measured by chemiluminescent enzyme immunoassay (Fujirebio, Tokyo, Japan). Insulin resistance was evaluated by homeostasis model assessment for insulin resistance (HOMA-IR) [14]. Hemoglobin A1c (HbA1c) was measured by latex agglutination method (Fujirebio) and expressed as the National Glycohemoglobin Standardization Program (NGSP) value. Free fatty acid (FFA; Eiken Chemical, Tokyo, Japan) and lactate (Kyowa Medex, Tokyo, Japan) were measured enzymatically. Total cholesterol (TC) was measured enzymatically (Sysmex, Hyogo, Japan). HDL-C was measured by a direct method (Fujirebio). Low-density lipoprotein-cholesterol (LDL-C) was calculated by the 
Friedewald formula. TG was measured enzymatically (Sekisui Medical, Tokyo, Japan). Remnant-like particle-TG (RLP-TG) was measured by an immunosorbent assay (Otsuka Pharmaceutical, Tokyo, Japan). Remnant lipoprotein-cholesterol (RemL-C) was measured with a homogeneous assay (MetaboRead ${ }^{\mathrm{TM}}$, Kyowa Medex). The TG concentration in the TRL was estimated using a homogeneous assay (VLDL-TG ${ }^{\mathrm{TM}}$, Shino-test, Tokyo, Japan) and represented as hepatic triglyceride-rich lipoprotein-triglyceride (hTRL-TG) $[12,15]$. apoAI, AII, B, CII, CIII, and E were measured by an immunoturbidimetric method (Sekisui Medical). apoB48 was measured by chemiluminescent enzyme immunoassay (Fujirebio). apoB100 levels were calculated by subtracting the value of apoB48 from the value of serum apoB [16]. The apoE phenotype was measured using the isometric electrophoresis method (Phenotyping ApoE IEF System ${ }^{\mathrm{TM}}$, Joko, Tokyo, Japan).

\subsection{Quantification of Postprandial Metabolism}

Postprandial metabolism was quantified by calculating the incremental area under the curve ( $\triangle \mathrm{AUC}$ ), which was defined as the difference between the area under the curve and the area below baseline $(0 \mathrm{~h})$ from $0 \mathrm{~h}$ to $6 \mathrm{~h}$, as described previously [11]. Postprandial changes in the concentrations of TG, RLP-TG, RemL-C, hTRL-TG, and apoB48 were calculated as the difference from the baseline mean value (as 0 at $0 \mathrm{~h}$ ), and represented as $\triangle \mathrm{TG}, \triangle \mathrm{RLP}-\mathrm{TG}, \Delta \mathrm{RemL}-\mathrm{C}$, $\triangle \mathrm{hTRL}-\mathrm{TG}$, and $\Delta$ apoB48, respectively.

\subsection{Statistics}

Statistical analyses were performed using the SPSS ver 23.0 (IBM, Tokyo, Japan). Because data were non-normally distributed by the Shapiro-Wilk test, all data were expressed as median and quartiles 1 and $3(\mathrm{Q} 1, \mathrm{Q} 3)$. Differences in the time-course compared with the fasting values in each trial were analyzed using Friedman test, followed by the Dunnett test. The measured value differences at each time-point in the 4 trials were assessed by the Schaffer test. $p<0.05$ was considered statistically significant.

\section{Results}

\section{1. $\operatorname{Exp} 1$}

The physical characteristics and fasting blood chemical data of the subjects in Exp 1 are shown in Table 1. There were no significant differences in any of them in the 4 trials (data not shown).

Table 1. Anthropometric and clinical characteristics in Exp 1 and 2.

\begin{tabular}{|c|c|c|c|c|}
\hline & Exp 1 & & $\operatorname{Exp} 2$ & \\
\hline Age (years) & 21.5 & $(21.0,22.0)$ & 21.5 & $(21.0,22.0)$ \\
\hline Height (cm) & 156.3 & $(152.4,159.0)$ & 158.6 & $(154.7,160.5)$ \\
\hline Body mass (kg) & 49.5 & $(44.9,52.7)$ & 52.2 & $(47.3,57.5)$ \\
\hline Waist (cm) & 72.0 & $(69.0,75.8)$ & 70.8 & $(64.8,75.5)$ \\
\hline $\mathrm{W} / \mathrm{H}$ & 0.80 & $(0.76,0.81)$ & 0.79 & $(0.75,0.80)$ \\
\hline $\operatorname{VFA}\left(\mathrm{cm}^{2}\right)$ & 28.1 & $(15.6,34.7)$ & 32.0 & $(20.8,47.8)$ \\
\hline $\mathrm{DBP}(\mathrm{mmHg})$ & 61.7 & $(59.0,67.0)$ & 64.0 & $(58.5,71.3)$ \\
\hline HbAlc (\%) & 5.2 & $(5.1,5.3)$ & 5.1 & $(5.0,5.5)$ \\
\hline HOMA-IR & 1.0 & $(0.8,1.4)$ & 1.1 & $(0.9,1.7)$ \\
\hline $\mathrm{TC}(\mathrm{mg} / \mathrm{dL})$ & 160.5 & $(138.8,180.3)$ & 176.5 & $(170.5,190.3)$ \\
\hline HDL-C (mg/dL) & 61.0 & $(50.5,67.3)$ & 68.5 & $(59.5,74.5)$ \\
\hline LDL-C (mg/dL) & 85.6 & $(75.9,96.7)$ & 98.9 & $(85.9,106.4)$ \\
\hline apoAI (mg/dL) & 148.5 & $(120.8,155.8)$ & 152.0 & $(139.0,165.8)$ \\
\hline apoAII (mg/dL) & 24.5 & $(22.3,27.9)$ & 25.1 & $(24.3,27.2)$ \\
\hline apoCIII (mg/dL) & 6.8 & $(5.2,7.3)$ & 7.2 & $(6.1,7.9)$ \\
\hline apoE (mg/dL) & 3.4 & $(3.2,4.1)$ & 4.4 & $(4.0,5.0)$ \\
\hline
\end{tabular}

All values are presented as median (Q1, Q3).

The serum concentrations of fasting and postprandial glucose, fructose, insulin, lactate, FFA, TG, RLP-TG, RemL-C, hTRL-TG, apoB100, and apoB48 in the 4 trials are presented in Table 2. In Figure 2, $\Delta \mathrm{TG}(\mathrm{A}), \triangle \mathrm{RLP}-\mathrm{TG}(\mathrm{C})$, $\Delta$ RemL-C (E), $\Delta$ hTRL-TG (G), and $\triangle$ apoB48 (I) are presented.

Compared with the respective baseline concentrations, the serum concentration of glucose significantly increased at 0.5 and $1 \mathrm{~h}$ in the $\mathrm{G}$ and $\mathrm{S}$ trials, and at $0.5 \mathrm{~h}$ in other 2 trials $(p<$ 0.05 each), whereas it decreased at 4 and $6 \mathrm{~h}$ in the $\mathrm{F}$ trial ( $p$ $<0.05$ each). The glucose concentration at $0.5 \mathrm{~h}$ in the $\mathrm{F}$ trial was significantly lower than that in other 3 trials $(p<0.05$ each). At $1 \mathrm{~h}$, it was higher in the $\mathrm{G}$ trial than in the $\mathrm{F}$ and GF trials $(p<0.05$ each). $\triangle$ AUC of glucose was not different among the 4 trials (data not shown).

Compared with the respective baseline concentrations, the fructose concentration significantly increased at $0.5,1$, and $2 \mathrm{~h}$ in the $\mathrm{F}$ trial, and at 0.5 and $1 \mathrm{~h}$ in the $\mathrm{S}$ and GF trials $(p<0.05$ each). The fructose concentration at 1 and $2 \mathrm{~h}$ was significantly higher in the $\mathrm{F}$ trial than in other 3 trials $(p<0.05$ each). The serum concentration of fructose did not significantly change during the experiment in the $G$ trial. $\triangle \mathrm{AUC}$ of fructose in the $\mathrm{F}$ trial was larger compared with the other 3 trials, and it was larger in the $\mathrm{S}$ trial compared with 
the G and GF trials ( $p<0.05$ each) (data not shown).

The serum concentration of insulin significantly increased at 0.5 and $1 \mathrm{~h}$ in the $\mathrm{G}$ and $\mathrm{S}$ trials, and at $0.5 \mathrm{~h}$ in the $\mathrm{F}$ and GF trials compared with the respective baseline concentrations ( $p$ $<0.05$ each). At $0.5 \mathrm{~h}$, the insulin concentration in the $\mathrm{F}$ trial was significantly lower than that in other 3 trials. At $1 \mathrm{~h}$, it was lower in the GF trial than that in the $\mathrm{G}$ and $\mathrm{S}$ trials, and in the $\mathrm{F}$ trial, lower than that in the G trial ( $p<0.05$ each). $\triangle \mathrm{AUC}$ of insulin in the $G$ trial was larger than those in other 3 trials, and in the $\mathrm{S}$ trial, it was larger than those in the $\mathrm{F}$ and GF trials ( $p<0.05$ each) (data not shown).

The serum concentration of lactate in the F, S, and GF trials increased at $1 \mathrm{~h}$ compared with the respective baseline concentrations ( $p<0.05$ each). In the $\mathrm{G}$ trial, no change was observed during the experiment. At $1 \mathrm{~h}$, the lactate concentration in the $\mathrm{G}$ trial was lower compared to the $\mathrm{F}$ and $\mathrm{S}$ trials ( $p<0.05$ each).

The serum concentration of FFA in the $G$ and $S$ trials decreased at 1 and $2 \mathrm{~h}$ and increased at 4 and $6 \mathrm{~h}$ compared with the respective baseline concentrations ( $p<0.05$ each). In the $\mathrm{F}$ trial, it decreased at $1 \mathrm{~h}$, and increased at $6 \mathrm{~h}$, and in the GF trial, it decreased at $1 \mathrm{~h}$ and increased at 4 and $6 \mathrm{~h}$ compared to each baseline ( $p<0.05$ each).

The serum concentrations of TG, RLP-TG, RemL-C, hTRL-TG, apoB100, and apoB48 did not significantly change in all trials during the experiment. However, in the $\mathrm{F}$ trial, non-significant increases of serum TG, RLP-TG, RemL-C, and hTRL-TG were observed.

Table 2. Parameters before and after beverage ingestion in Exp 1.

\begin{tabular}{|c|c|c|c|c|c|c|c|c|}
\hline & Trial & & $\mathbf{0 ~ h}$ & & $0.5 \mathrm{~h}$ & & $1 \mathrm{~h}$ & \\
\hline \multirow{4}{*}{ Glucose (mg/dL) } & $\mathrm{G}$ & 86.1 & $(80.1,88.4)$ & 123.4 & $\left.(117.1,140.4)^{*}\right]_{+}$ & 103.7 & $(87.4,129.2)^{*}$ & \\
\hline & $\mathrm{F}$ & 84.3 & $(82.6,85.7)$ & 91.2 & (88.2, 94.4)* & 84.9 & $(81.6,86.8)$ & \\
\hline & $\mathrm{S}$ & 81.7 & $(79.5,86.8)$ & 120.9 & $\left.(114.4,130.1)^{*}\right]\left.^{\dagger}\right|_{\dagger}$ & 92.1 & $(75.4,99.3)^{*}$ & $\dagger$ \\
\hline & GF & 83.8 & $(80.9,87.6)$ & 106.7 & $\left.(103.8126 .5)^{*}\right\rfloor$ & 84.1 & $(71.5,92.3)$ & \\
\hline \multirow{4}{*}{ Fructose $(\mathrm{mg} / \mathrm{dL})$} & $\mathrm{G}$ & 0.63 & $(0.46,0.79)$ & 0.65 & $(0.20,0.74)$ & 0.56 & $(0.34,0.71)$ & \\
\hline & $\mathrm{F}$ & 0.61 & $(0.45,0.82)$ & 8.41 & $(5.54,9.72)^{*}$ & 6.00 & $(5.76,8.43)^{*}$ & \\
\hline & $\mathrm{S}$ & 0.56 & $(0.36,0.68)$ & 4.88 & $(3.46,5.89)^{*}$ & 2.81 & $(1.89,3.71)^{*}$ & \\
\hline & GF & 0.69 & $(0.42,0.85)$ & 4.61 & $\left.(3.38,5.90)^{*}\right]^{\dagger}$ & 2.28 & $(1.84,2.96)^{*}$ & ]$\left.^{\dagger}\right]^{\dagger}$ \\
\hline \multirow{4}{*}{ Insulin $(\mu \mathrm{U} / \mathrm{mL})$} & $\bar{G}$ & 4.9 & $(4.0,6.2)$ & 36.8 & $(27.7,46.8)^{*}$ & 20.0 & $(14.6,23.4)^{*}$ & $7+$ \\
\hline & $\mathrm{F}$ & 7.3 & $(5.9,8.6)$ & 11.5 & $(9.7,19.4)^{*}$ & 8.3 & $(7.5,11.8)$ & \\
\hline & $\mathrm{S}$ & 5.7 & $(4.8,6.7)$ & 29.0 & $(23.2,36.5)^{*}$ & 14.0 & $(7.2,17.1)^{*}$ & \\
\hline & GF & 6.0 & $(4.7,7.7)$ & 24.9 & $\left.\left.(14.5,35.9)^{*}\right]^{\dagger}\right]$ & 7.3 & $(6.1,9.1)$ & $\dagger$ \\
\hline \multirow{4}{*}{ Lactate (mg/dL) } & $\bar{G}$ & 10.1 & $(8.7,12.6)$ & & & 12.7 & $(7.2,16.8)$ & \\
\hline & $\mathrm{F}$ & 11.4 & $(8.5,12.8)$ & & & 18.1 & $(15.0,21.3)^{*}$ & \\
\hline & $\mathrm{S}$ & 10.1 & $(8.7,11.6)$ & & & 17.0 & $(13.8,18.8)^{*}$ & \\
\hline & GF & 10.8 & $(8.3,12.2)$ & & & 16.2 & $(10.6,17.6)^{*}$ & \\
\hline \multirow{4}{*}{$\mathrm{FFA}(\mathrm{mmol} / \mathrm{L})$} & $\bar{G}$ & 0.39 & $(0.31,0.53)$ & & & 0.09 & $(0.05,0.11)^{*}$ & $\dagger$ \\
\hline & F & 0.41 & $(0.33,0.57)$ & & & 0.16 & $(0.12,0.18)^{*}$ & \\
\hline & $\mathrm{S}$ & 0.45 & $(0.27,0.61)$ & & & 0.10 & $(0.07,0.13)^{*}$ & \\
\hline & GF & 0.47 & $\left(\begin{array}{ll}0.38 & 0.51\end{array}\right)$ & & & 0.10 & $(0.08,0.15)^{*}$ & \\
\hline \multirow{4}{*}{$\mathrm{TG}(\mathrm{mg} / \mathrm{dL})$} & $\mathrm{G}$ & 56.0 & $(39.5,67.0)$ & & & 55.0 & $(44.8,67.3)$ & \\
\hline & $\mathrm{F}$ & 51.0 & $(46.0,69.0)$ & & & 51.5 & $(45.3,66.0)$ & \\
\hline & $\mathrm{S}$ & 49.5 & $(40.0,71.8)$ & & & 46.0 & $(41.3,65.3)$ & \\
\hline & GF & 49.0 & $(39.5,63.5)$ & & & 51.0 & $(38.8,62.0)$ & \\
\hline \multirow{4}{*}{ RLP-TG (mg/dL) } & G & 8.7 & $(6.7,9.7)$ & & & 10.2 & $(7.9,11.1)$ & \\
\hline & F & 7.9 & $(7.2,13.7)$ & & & 8.5 & $(7.5,11.7)$ & \\
\hline & $\mathrm{S}$ & 8.3 & $(6.7,13.0)$ & & & 9.7 & $(7.2,13.4)$ & \\
\hline & GF & 8.1 & $(7.2,9.6)$ & & & 9.2 & $(6.7,11.0)$ & \\
\hline \multirow{4}{*}{ RemL-C (mg/dL) } & $\bar{G}$ & 4.05 & $(2.68,4.80)$ & & & 3.95 & $(2.63,4.78)$ & \\
\hline & $\mathrm{F}$ & 3.40 & $(3.00,5.05)$ & & & 3.15 & $(2.95,4.73)$ & \\
\hline & $\mathrm{S}$ & 3.95 & $(2.70,4.53)$ & & & 3.45 & $(2.80,4.10)$ & \\
\hline & GF & 3.35 & $(2.28,4.18)$ & & & 3.55 & $(2.08,4.03)$ & \\
\hline \multirow{4}{*}{$\mathrm{hTRL}-\mathrm{TG}(\mathrm{mg} / \mathrm{dL})$} & $\bar{G}$ & 45.0 & $(27.8,54.0)$ & & & 44.5 & $(31.5,54.5)$ & \\
\hline & $\mathrm{F}$ & 37.0 & $(32.0,57.5)$ & & & 38.5 & $(33.0,56.8)$ & \\
\hline & $\mathrm{S}$ & 35.5 & $(28.5,60.5)$ & & & 34.0 & $(30.0,54.5)$ & \\
\hline & GF & 39.0 & $(27.0,52.0)$ & & & 40.0 & $(26.3,49.8)$ & \\
\hline \multirow{4}{*}{ apoB100 (mg/dL) } & $\bar{G}$ & 61.9 & $(56.9,68.1)$ & & & 60.7 & $(55.6,66.4)$ & \\
\hline & $\mathrm{F}$ & 62.8 & $(57.9,68.2)$ & & & 62.3 & $(54.9,66.9)$ & \\
\hline & $\mathrm{S}$ & 64.8 & $(60.3,68.1)$ & & & 62.2 & $(56.9,66.3)$ & \\
\hline & GF & 60.3 & $(58.6,73.3)$ & & & 59.9 & $(55.9,71.8)$ & \\
\hline \multirow{4}{*}{ apoB48 $(\mu \mathrm{g} / \mathrm{mL})$} & G & 1.40 & $(1.18,2.55)$ & & & 2.25 & $(1.70,3.35)$ & \\
\hline & $\mathrm{F}$ & 1.60 & $(1.13,2.03)$ & & & 1.40 & $(1.08,1.70)$ & \\
\hline & $\mathrm{S}$ & 1.65 & $(1.40,1.95)$ & & & 1.95 & $(1.43,2.80)$ & \\
\hline & GF & 1.55 & $(1.23,1.93)$ & & & 2.05 & $(1.45,2.38)$ & \\
\hline
\end{tabular}


Table 2. Continued.

\begin{tabular}{|c|c|c|c|c|c|c|c|c|}
\hline & Trial & & $2 \mathrm{~h}$ & & & $4 \mathrm{~h}$ & & $6 \mathrm{~h}$ \\
\hline \multirow{4}{*}{ Glucose (mg/dL) } & $\mathrm{G}$ & 82.0 & $(68.6,87.2)$ & & 78.5 & $(76.2,80.5)$ & 79.1 & $(76.3,83.7)$ \\
\hline & $\mathrm{F}$ & 83.7 & $(80.1,85.1)$ & \multirow{3}{*}{ 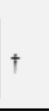 } & 77.9 & $(76.7,80.2)^{*}$ & 77.6 & $(76.6,81.9)^{*}$ \\
\hline & $\mathrm{S}$ & 79.4 & $(75.6,82.0)$ & & 81.6 & $(77.4,83.9)$ & 78.7 & $(77.0,81.1)$ \\
\hline & GF & 80.1 & $(77.6,83.1)$ & & 78.8 & $(77.1,81.0)$ & 77.4 & $(74.3,78.9)$ \\
\hline \multirow{4}{*}{ Fructose (mg/dL) } & $\bar{G}$ & 0.65 & $(0.47,0.86)$ & \multirow{3}{*}{ - } & 0.43 & $(0.26,0.66)$ & 0.36 & $(0.11,0.71)$ \\
\hline & $\mathrm{F}$ & 1.84 & $(1.38,3.14)^{*}$ & & 0.79 & $(0.71,0.91)$ & 0.47 & $(0.13,0.65)$ \\
\hline & $\mathrm{S}$ & 1.10 & $(0.88,1.37)$ & & 0.68 & $(0.41,1.31)$ & 0.61 & $(0.23,1.16)$ \\
\hline & GF & 0.99 & $(0.77,1.26)$ & 1 & 0.63 & $(0.34,1.26)$ & 0.63 & $(0.11,1.27)$ \\
\hline \multirow{4}{*}{ In sulin $(\mu \mathrm{U} / \mathrm{mL})$} & $\bar{G}$ & 5.2 & $(2.5,6.4)$ & \multirow{4}{*}{$\dagger$} & 2.9 & $(2.5,3.2)$ & 3.0 & $(2.6,4.7)$ \\
\hline & $\mathrm{F}$ & 6.2 & $(4.4,7.9)$ & & 3.7 & $(3.4,5.5)$ & 2.8 & $(2.3,3.9)^{*}$ \\
\hline & $\mathrm{S}$ & 4.8 & $(4.1,5.9)$ & & 3.9 & $(3.0,4.6)$ & 3.4 & $(2.7,3.9)$ \\
\hline & GF & 3.6 & $(2.9,6.4)$ & & 2.8 & $\left(\begin{array}{ll}2.2 & 4.0\end{array}\right)$ & 2.9 & $(1.6,3.5)$ \\
\hline \multirow{4}{*}{ Lactate $(\mathrm{mg} / \mathrm{dL})$} & $\mathrm{G}$ & 9.4 & $(6.6,12.5)$ & & 8.9 & $(6.7,10.7)$ & 8.9 & $(6.9,10.2)$ \\
\hline & $\mathrm{F}$ & 10.2 & $(7.4,13.9)$ & & 8.3 & $(6.9,9.9)$ & 8.4 & $(7.2,10.1)$ \\
\hline & $\mathrm{S}$ & 10.3 & $(8.6,11.0)$ & & 8.2 & $(6.7,8.9)$ & 8.4 & $(7.2,9.9)$ \\
\hline & GF & 9.4 & $(6.3,11.4)$ & & 9.5 & $(7.3,10.2)$ & 9.0 & $(6.2,10.3)$ \\
\hline \multirow{4}{*}{$\mathrm{FFA}(\mathrm{mmol} / \mathrm{L})$} & $\bar{G}$ & 0.18 & $(0.09,0.28)^{*}$ & & 0.59 & $(0.51,0.69)^{*}$ & 0.87 & $(0.74,0.94)^{*}$ \\
\hline & $\mathrm{F}$ & 0.32 & $(0.24,0.37)$ & & 0.59 & $(0.43,0.69)$ & 0.68 & $(0.58,0.84)^{*}$ \\
\hline & $\mathrm{S}$ & 0.24 & $(0.20,0.35)^{*}$ & & 0.61 & $(0.49,0.77)^{*}$ & 0.80 & $(0.72,0.81)^{*}$ \\
\hline & GF & 0.37 & $(0.24,0.54)$ & & 0.61 & $(0.50,0.75)^{*}$ & 0.76 & $(0.68,1.00)^{*}$ \\
\hline \multirow{4}{*}{$\mathrm{TG}(\mathrm{mg} / \mathrm{dL})$} & $\bar{G}$ & 48.5 & $(40.3,57.8)$ & & 59.0 & $(48.5,63.8)$ & 59.5 & $(53.5,74.5)$ \\
\hline & $\mathrm{F}$ & 51.0 & $(44.0,68.3)$ & & 62.0 & $(55.8,75.8)$ & 62.0 & $(57.8,80.0)$ \\
\hline & $\mathrm{S}$ & 43.5 & $(38.0,59.3)$ & & 52.0 & $(48.5,72.0)$ & 54.5 & $(46.8,69.0)$ \\
\hline & GF & 46.5 & $(35.3,61.0)$ & & 55.5 & $(41.3,74.3)$ & 56.5 & $(41.8,71.0)$ \\
\hline \multirow{4}{*}{ RLP-TG (mg/dL) } & G & 7.6 & $(6.1,8.1)$ & & 7.9 & $(6.4,8.4)$ & 8.2 & $(5.7,10.6)$ \\
\hline & $\mathrm{F}$ & 8.8 & $(6.6,11.3)$ & & 8.5 & $(7.0,12.1)$ & 9.9 & $(8.0,12.4)$ \\
\hline & $\mathrm{S}$ & 8.2 & $(6.2,11.4)$ & & 9.0 & $(6.9,12.4)$ & 9.4 & $(7.3,11.6)$ \\
\hline & GF & 7.1 & $(5.8,8.4)$ & & 8.8 & $(6.5,10.4)$ & 8.9 & $(7.0,9.4)$ \\
\hline \multirow{4}{*}{ RemL-C (mg/dL) } & $\mathrm{G}$ & 3.40 & $(2.38,4.43)$ & & 3.50 & $(2.88,4.73)$ & 4.10 & $(3.63,5.45)$ \\
\hline & $\mathrm{F}$ & 3.15 & $(2.75,4.55)$ & & 3.90 & $(3.38,5.30)$ & 4.45 & $(3.70,5.75)$ \\
\hline & $\mathrm{S}$ & 3.20 & $(2.75,3.80)$ & & 3.55 & $(3.00,4.40)$ & 3.85 & $(3.53,4.48)$ \\
\hline & GF & 3.30 & $(1.98,3.88)$ & & 3.85 & $(2.28,4.70)$ & 4.05 & $(2.58,4.90)$ \\
\hline \multirow{4}{*}{ hTRL-TG (mg/dL) } & $\mathrm{G}$ & 38.0 & $(26.3,46.5)$ & & 50.5 & $(35.3,52.8)$ & 48.0 & $(41.5,64.3)$ \\
\hline & $\mathrm{F}$ & 38.5 & $(34.3,61.3)$ & & 51.5 & $(45.5,71.8)$ & 54.5 & $(42.5,72.3)$ \\
\hline & $\mathrm{S}$ & 32.5 & $(26.5,48.8)$ & & 42.0 & $(37.8,64.0)$ & 45.0 & $(37.5,63.3)$ \\
\hline & GF & 36.5 & $(23.5,46.3)$ & & 48.0 & $(30.8,60.8)$ & 47.5 & $(32.0,57.5)$ \\
\hline \multirow{4}{*}{ apoB100 (mg/dL) } & $\bar{G}$ & 60.3 & $(56.5,66.6)$ & & 61.4 & $(57.3,68.0)$ & 61.4 & $(57.9,69.9)$ \\
\hline & $\mathrm{F}$ & 62.3 & $(56.6,65.9)$ & & 63.2 & $(56.6,67.1)$ & 63.3 & $(57.6,68.0)$ \\
\hline & $\mathrm{S}$ & 62.8 & $(58.3,67.5)$ & & 65.4 & $(59.6,67.6)$ & 64.9 & $(59.6,71.3)$ \\
\hline & GF & 58.9 & $(56.6,71.3)$ & & 65.4 & $(57.6,73.6)$ & 60.8 & $(57.6,73.5)$ \\
\hline \multirow{4}{*}{ apoB48 $(\mu \mathrm{g} / \mathrm{mL})$} & $\bar{G}$ & 1.95 & $(1.65,2.65)$ & & 1.15 & $(0.95,1.58)$ & 1.15 & $(0.98,1.45)$ \\
\hline & $\mathrm{F}$ & 1.65 & $(1.33,2.28)$ & & 1.80 & $(1.50,2.08)$ & 1.85 & $(1.68,2.43)$ \\
\hline & $\mathrm{S}$ & 1.90 & $(1.25,2.22)$ & & 1.35 & $(1.20,1.60)$ & 1.45 & $(1.08,2.03)$ \\
\hline & GF & 1.70 & $(1.43,2.18)$ & & 1.50 & $(0.88,1.70)$ & 1.60 & $(1.25,2.20)$ \\
\hline
\end{tabular}

All values are presented as median (Q1, Q3). ${ }^{*} p<0.05$ compared to the fasting values. ${ }^{\dagger} p<0.05$ compared between the trials.

\section{2. $\operatorname{Exp} 2$}

The physical characteristics and fasting blood chemical data of the subjects in Exp 2 are shown in Table 1. There were no significant differences in any of them in the 4 trials (data not shown). The concentrations of serum fasting and postprandial glucose, fructose, insulin, lactate, FFA, TG, RLP-TG, RemL-C, hTRL-TG, apoB100, and apoB48 in the 4 trials are presented in Table 3. $\triangle \mathrm{AUC}$ of these parameters are shown in Table 4. $\Delta \mathrm{TG}$ (B), $\Delta$ RLP-TG (D), $\Delta$ RemL-C (F), $\triangle \mathrm{hTRL}-\mathrm{TG}(\mathrm{H})$, and $\triangle \mathrm{apoB} 48(\mathrm{~J})$ are presented in Figure 2.

The serum concentration of glucose increased at $0.5 \mathrm{~h}$ in the GFt and SFt trials. In the FFt and Ft trials, the glucose concentration did not significantly change during the experiment.

The serum concentration of fructose significantly increased at $0.5,1$, and $2 \mathrm{~h}$ in the FFt trial, and at 0.5 and $1 \mathrm{~h}$ in the SFt trial compared with the respective baseline concentrations $(p$ $<0.05$ each). The fructose concentration at $0.5,1$, and $2 \mathrm{~h}$ in the FFt trial was higher than that in the SFt trial $(p<0.05$ each). The fructose concentration did not change significantly in the GFt and Ft trials during the experiment. $\triangle \mathrm{AUC}$ of fructose was larger in the FFt trial compared to other 3 trials and, in the SFt trial, it was larger than in the GFt and Ft trials.

Compared with the respective baseline concentrations, the serum concentration of insulin significantly increased at 0.5 and $1 \mathrm{~h}$ in the GFt and SFt trials ( $p<0.05$ each). The insulin concentration at $0.5 \mathrm{~h}$ in the FFt trial was significantly lower 
than that in the GFt and SFt trials $(p<0.05)$. The insulin concentration did not change significantly in the FFt and $\mathrm{Ft}$ trilas during the experiment. $\triangle \mathrm{AUC}$ of insulin was smaller in the FFt trial compared to the GFt and SFt trials.
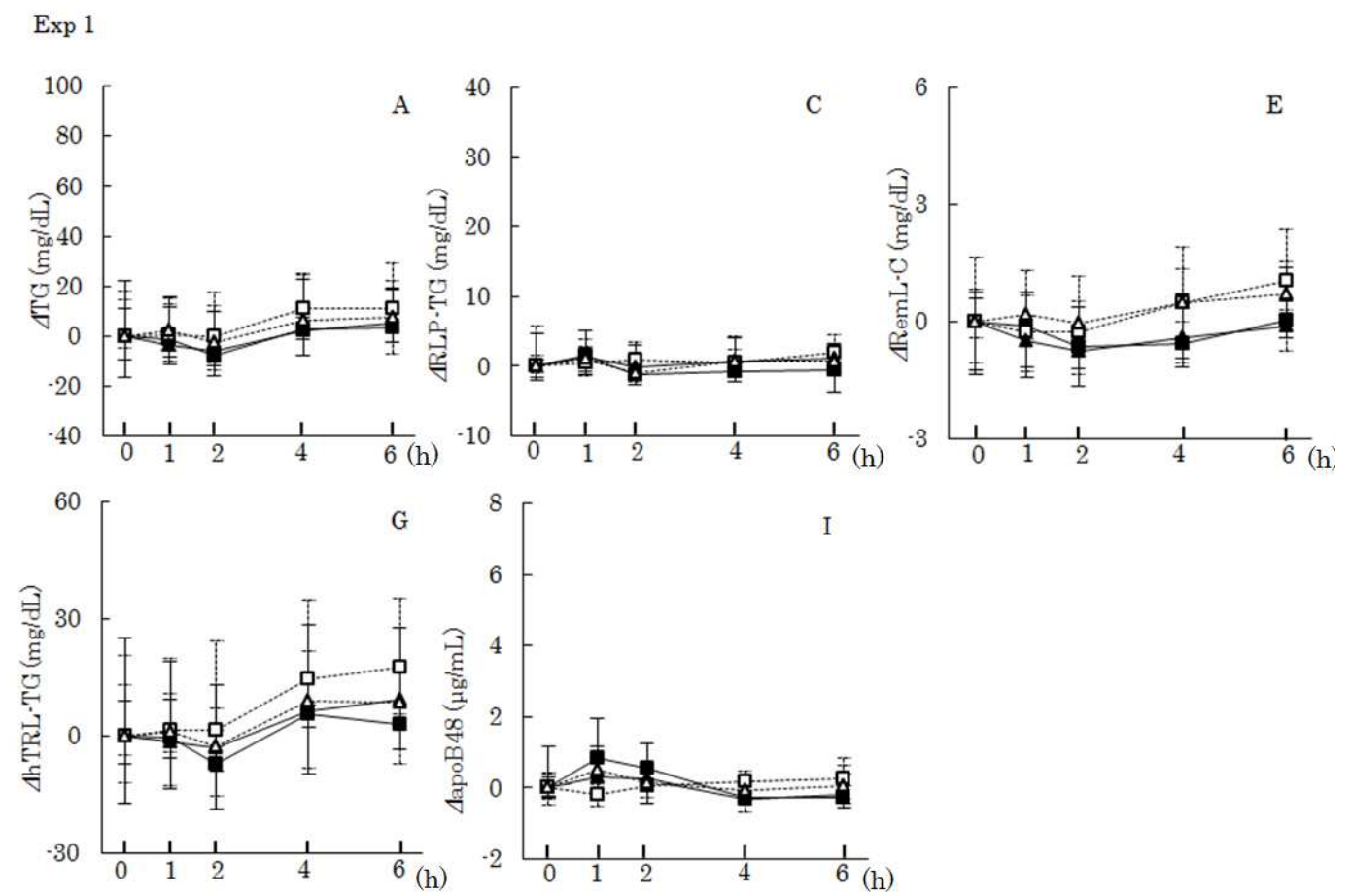

$\operatorname{Exp} 2$
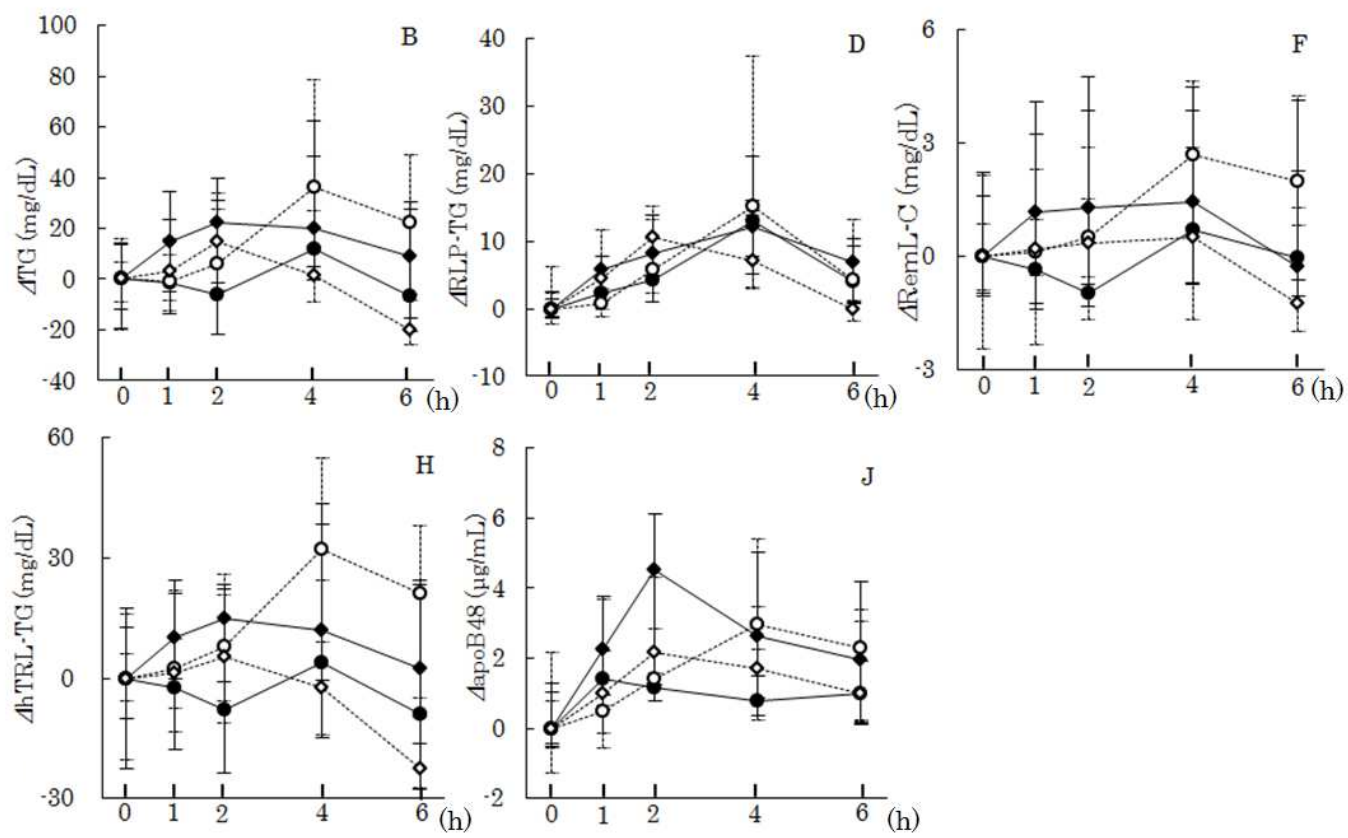

Figure 2. Postprandial changes of $\triangle T G$ ( $A$ and $B), \triangle R L P-T G(C$ and D), $\triangle R e m L-C$ (E and F), $\triangle h T R L-T G$ (G and H), and $\triangle a p o B 48$ (I and J) in Exp 1 and 2.

Exp $1 \mathbf{\mathbf { m }}: \mathrm{G}, \square: \mathrm{F}, \boldsymbol{\Delta}: \mathrm{S}, \Delta: \mathrm{GF}$

Exp $2 \bullet: \mathrm{GFt}, \circ: \mathrm{FFt}, \diamond: \mathrm{SFt}, \diamond: \mathrm{Ft}$

Data are expressed as median and quartiles 1 and 3 (Q1, Q3).

The serum concentration of lactate significantly increased at $1 \mathrm{~h}$ in the FFt and SFt trials, and significantly decreased at 6 $\mathrm{h}$ in the GFt trial compared with the respective baseline concentrations ( $p<0.05$ each). The lactate concentration did not significantly change in the Ft trial during the experiment.
The serum concentration of FFA significantly decreased at 1 $\mathrm{h}$ in the GFt, FFt, and SFt trials compared with the respective baseline concentrations. It increased at $4 \mathrm{~h}$ in the $\mathrm{SFt}$ trial, and at $6 \mathrm{~h}$ in the trials except the FFt trial ( $p<0.05$ each). $\triangle \mathrm{AUC}$ of FFA was smaller in the FFt trial compared with th SFt and 
Ft trials $(p<0.05$ each). $\triangle \mathrm{AUC}$ in the Ft trial tended to be larger than that in GFt and SFt trials $(p<0.1$ each).

The serum concentration of $\mathrm{TG}$ did not increase significantly except at $4 \mathrm{~h}$ in the FFt trial compared with the respective baseline concentrations (Figure 2B). The TG concentration peaked at $2 \mathrm{~h}$ in the Ft and SFt trials, and at $4 \mathrm{~h}$ in other 2 trials. $\triangle \mathrm{AUC}$ in the FFt trial tended to be larger than that in the GFt $(p<0.05)$ and Ft $(p<0.1)$ trials.

The RLP-TG concentration significantly increased at $4 \mathrm{~h}$ in the FFt trial; at 1 and $2 \mathrm{~h}$ in the SFt trial; and at 2 and $4 \mathrm{~h}$ in the Ft trial compared with the respective baseline concentrations ( $p<0.05$ each). In the GFt trial, the change of RLP-TG concentration was not significant during the experiment. The RLP-TG concentration peaked at $2 \mathrm{~h}$ in the Ft trial and at $4 \mathrm{~h}$ in other 3 trials (Figure 2D).

The RemL-C concentration significantly increased at 4 and 6 $\mathrm{h}$ in the FFt trial compared with the baseline concentration $(p<$
0.05 each; Figure 2F). The RemL-C concentration did not change significantly during the experiment in other 3 trials.

The hTRL-TG concentration increased at $4 \mathrm{~h}$ in the FFt trial compared with the baseline concentration $(p<0.05$; Figure $2 \mathrm{H})$. The hTRL-TG concentration peaked at $2 \mathrm{~h}$ in the Ft trial and at $4 \mathrm{~h}$ in the FFt and SFt trials. There was no significant change during the $6 \mathrm{~h}$ in other 3 trials. $\triangle \mathrm{AUC}$ was larger in the FFt trial compared with the GFt and Ft trials, and in the SFt trial, it was larger than that in the GFt trial.

The serum concentration of apoB100 did not change significantly in all trials. Compared with the respective baseline concentrations, the concentration of apoB48 significantly increased at 1 and $2 \mathrm{~h}$ in the GFt trial; at 2, 4, and $6 \mathrm{~h}$ in the FFt trial; and at 2 and $4 \mathrm{~h}$ in the SFt trial ( $p<0.05$ each; Figure $2 \mathrm{~J}$ ). The apoB48 concentration peaked at $1 \mathrm{~h}$ in the GFt trial; at $2 \mathrm{~h}$ in the Ft and SFt trials; and at $4 \mathrm{~h}$ in the FFt trial.

Table 3. Parameters before and after beverage ingestion in Exp 2.

\begin{tabular}{|c|c|c|c|c|c|c|c|c|}
\hline & Trial & & $\mathbf{0 ~ h}$ & & $0.5 \mathrm{~h}$ & & $1 \mathrm{~h}$ & \\
\hline \multirow{4}{*}{ Glucose (mg/dL) } & GFt & 73.0 & $(64.7,81.5)$ & 114.9 & $\left.(93.8,126.4)^{*}\right]+$ & 83.6 & $(71.3,115.8)$ & \\
\hline & FFt & 75.5 & $(70.1,78.9)$ & 77.4 & $(74.9,86.2)$ & 76.8 & $(67.1,85.0)$ & \\
\hline & SFt & 76.6 & $(69.0,81.1)$ & 110.9 & $(97.9,120.8)^{*}$ & 75.9 & $(66.3,84.5)$ & \\
\hline & $\mathrm{Ft}$ & 73.6 & $(63.7,80.5)$ & 75.0 & $(67.7,86.8)$ & 77.7 & $(57.2,82.5)$ & \\
\hline \multirow{4}{*}{ Fructose (mg/dL) } & GFt & 0.63 & $(0.41,0.92)$ & 0.74 & $(0.46,0.91)$ & 0.61 & $(0.50,0.82)$ & \\
\hline & FFt & 0.79 & $(0.52,1.00)$ & 6.23 & $(4.85,7.39)^{*}$ & 5.42 & $(4.84,5.91)^{*}=$ & $\dagger$ \\
\hline & SFt & 0.88 & $(0.52,0.96)$ & 4.42 & $\left.(2.87,5.15)^{*}\right]^{\top}$ & 2.47 & $(2.16,3.20)^{*}=$ & \\
\hline & $\mathrm{Ft}$ & 0.79 & $\left(\begin{array}{ll}0.61 & 0.96\end{array}\right)$ & 0.69 & $(0.56,0.77) \quad]^{\dagger}$ & 0.67 & $(0.57,0.77)$ & \\
\hline \multirow{4}{*}{ Insulin $(\mu \mathrm{U} / \mathrm{mL})$} & GFt & 5.9 & $(5.1,9.3)$ & 42.7 & $\left.(36.7,66.4)^{*}\right]+7+$ & 25.0 & $(9.4,45.2)^{*}$ & \\
\hline & FFt & 6.0 & $(4.0,8.8)$ & 16.7 & $(9.2,18.0)$ & 13.2 & $(10.3,14.8)$ & \\
\hline & SFt & 5.6 & $(3.8,8.3)$ & 48.9 & $(37.7,58.8)^{*}$ & 13.5 & $(10.9,17.5)^{*}$ & \\
\hline & $\mathrm{Ft}$ & 6.7 & $(3.1,8.4)$ & 9.3 & $(6.6,13.6)$ & 8.3 & $(6.1,11.1)$ & \\
\hline \multirow{4}{*}{ Lactate $(\mathrm{mg} / \mathrm{dL})$} & GFt & 10.4 & $(8.8,12.2)$ & & & 12.0 & $(10.2,14.9)$ & \\
\hline & FFt & 10.2 & $(8.7,11.8)$ & & & 17.9 & $(15.0,21.3)^{*}$ & \\
\hline & SFt & 10.1 & $(8.2,13.0)$ & & & 19.1 & $(15.8,21.5)^{*}=$ & $\dagger$ \\
\hline & $\mathrm{Ft}$ & 11.8 & $(10.1,14.3)$ & & & 9.0 & $(6.6,12.3)$ & \\
\hline \multirow{4}{*}{ FFA $(\mathrm{mmol} / \mathrm{L})$} & GFt & 0.49 & $(0.37,0.60)$ & & & 0.15 & $(0.08,0.20)^{*}$ & \\
\hline & $\mathrm{FFt}$ & 0.46 & $(0.38,0.67)$ & & & 0.23 & $(0.13,0.26)^{*}$ & $\dagger$ \\
\hline & SFt & 0.42 & $(0.28,0.46)$ & & & 0.12 & $(0.08,0.20)^{*}$ & \\
\hline & $\mathrm{Ft}$ & 0.54 & $(0.37,0.68)$ & & & 0.42 & $(0.34,0.60)$ & \\
\hline \multirow{4}{*}{$\mathrm{TG}(\mathrm{mg} / \mathrm{dL})$} & GFt & 61.0 & $(41.5,74.8)$ & & & 59.5 & $(47.5,95.3)$ & \\
\hline & FFt & 51.0 & $(42.0,57.8)$ & & & 50.0 & $(42.5,60.3)$ & \\
\hline & SFt & 55.5 & $(43.5,69.8)$ & & & 70.5 & $(50.8,78.8)$ & \\
\hline & $\mathrm{Ft}$ & 62.0 & $(42.0,77.8)$ & & & 65.0 & $(49.8,78.0)$ & \\
\hline \multirow{4}{*}{ RLP-TG (mg/dL) } & GFt & 8.0 & $(6.8,10.6)$ & & & 10.3 & $(9.3,14.0)$ & \\
\hline & FFt & 9.1 & $(7.9,11.5)$ & & & 9.8 & $(7.9,12.0)$ & \\
\hline & SFt & 8.5 & $(7.2,10.0)$ & & & 14.3 & $(10.8,16.3)^{*}$ & \\
\hline & $\mathrm{Ft}$ & 9.7 & $(7.4,16.0)$ & & & 14.3 & $(9.6,21.3)$ & \\
\hline \multirow{4}{*}{ RemL-C (mg/dL) } & GFt & 4.10 & $(3.05,6.30)$ & & & 3.75 & $(2.83,8.18)$ & \\
\hline & $\mathrm{FFt}$ & 4.10 & $(3.18,4.95)$ & & & 4.20 & $(3.58,5.05)$ & \\
\hline & SFt & 5.05 & $(4.05,6.63)$ & & & 5.65 & $(4.08,7.30)$ & \\
\hline & $\mathrm{Ft}$ & 6.00 & $(3.53,8.15)$ & & & 6.20 & $(3.63,8.30)$ & \\
\hline \multirow{4}{*}{ hTRL-TG (mg/dL) } & GFt & 52.5 & $(30.0,65.3)$ & & & 50.0 & $(34.8,77.0)$ & \\
\hline & FFt & 39.0 & $(33.5,45.0)$ & & & 41.5 & $(31.5,48.8)$ & \\
\hline & SFt & 45.5 & $(35.3,63.0)$ & & & 55.5 & $(45.5,66.8)$ & \\
\hline & $\mathrm{Ft}$ & 52.0 & $(31.8,68.0)$ & & & 53.5 & $(38.8,74.0)$ & \\
\hline \multirow{4}{*}{ apoB100 (mg/dL) } & GFt & 70.9 & $(62.6,79.8)$ & & & 68.4 & $(60.7,78.2)$ & \\
\hline & $\mathrm{FFt}$ & 71.3 & $(57.1,78.3)$ & & & 70.3 & $(57.7,78.7)$ & \\
\hline & SFt & 69.4 & $(60.8,85.1)$ & & & 67.2 & $(59.5,81.3)$ & \\
\hline & $\mathrm{Ft}$ & 68.1 & $(55.6,79.2)$ & & & 69.1 & $(52.4,77.5)$ & \\
\hline \multirow{4}{*}{$\operatorname{apoB} 48(\mu \mathrm{g} / \mathrm{mL})$} & GFt & 1.40 & $(0.90,2.43)$ & & & 2.80 & $(1.95,3.63)^{*}$ & \\
\hline & FFt & 1.55 & $\left(\begin{array}{ll}0.98 & 2.33\end{array}\right)$ & & & 2.05 & $(0.98,2.23)$ & \\
\hline & SFt & 1.80 & $(1.38,3.08)$ & & & 4.05 & $(2.75,5.58)$ & \\
\hline & $\mathrm{Ft}$ & 2.55 & $(1.28,3.45)$ & & & 3.55 & $(2.40,5.08)$ & \\
\hline
\end{tabular}


Table 3. Continued.

\begin{tabular}{|c|c|c|c|c|c|c|c|c|}
\hline & Trial & & $2 \mathrm{~h}$ & & & $4 \mathrm{~h}$ & & $6 \mathrm{~h}$ \\
\hline \multirow{4}{*}{ Glucose (mg/dL) } & GFt & 72.7 & $(64.9,82.0)$ & & 74.2 & $(62.3,82.7)$ & 71.8 & $(57.5,77.1)$ \\
\hline & $\mathrm{FFt}$ & 75.9 & $(66.0,85.1)$ & & 71.6 & $(61.6,80.0)$ & 71.0 & $(62.1,79.0)$ \\
\hline & SFt & 76.3 & $(65.2,81.3)$ & & 72.8 & $(66.0,81.0)$ & 71.0 & $(61.8,79.6)$ \\
\hline & $\mathrm{Ft}$ & 78.2 & $(56.9,86.6)$ & & 75.9 & $(62.8,83.1)$ & 74.2 & $(59.3,77.8)$ \\
\hline \multirow{4}{*}{ Fructose $(\mathrm{mg} / \mathrm{dL})$} & GFt & 0.65 & $(0.48,0.72)$ & $T$ & 0.54 & $(0.44,0.72)$ & 0.45 & $(0.32,0.79)$ \\
\hline & $\mathrm{FFt}$ & 3.00 & $(2.42,3.29)^{*}=$ & $\dagger$ & 0.92 & $(0.71,1.08)$ & 0.56 & $(0.23,0.77)$ \\
\hline & SFt & 1.16 & $(0.97,1.58)$ & $\sqrt{t}$ & 0.44 & $(0.40,0.88)$ & 0.55 & $(0.42,0.63)$ \\
\hline & $\mathrm{Ft}$ & 0.54 & $(0.34,0.96)$ & & 0.72 & $(0.60,0.83)$ & 0.47 & $(0.41,0.74)$ \\
\hline \multirow{4}{*}{ Insulin $(\mu \mathrm{U} / \mathrm{mL})$} & GFt & 8.1 & $(6.7,9.8)$ & & 4.4 & $(3.4,5.3)$ & 3.1 & $(2.7,4.2)$ \\
\hline & $\mathrm{FFt}$ & 9.5 & $(6.1,14.0)$ & & 3.3 & $(2.7,7.3)$ & 4.1 & $(2.5,5.1)$ \\
\hline & SFt & 6.7 & $(5.5,9.3)$ & & 4.2 & $(3.1,5.7)$ & 2.9 & $(2.6,4.0)$ \\
\hline & $\mathrm{Ft}$ & 5.6 & $(4.1,7.0)$ & & 4.3 & $(3.1,5.4)$ & 3.1 & $(2.3,4.3)$ \\
\hline \multirow{4}{*}{ Lactate $(\mathrm{mg} / \mathrm{dL})$} & GFt & 9.5 & $(8.1,11.3)$ & & 7.3 & $(5.6,9.8)$ & 6.3 & $(5.3,8.1)^{*}$ \\
\hline & $\mathrm{FFt}$ & 12.1 & $(10.3,17.7)$ & & 7.9 & $(5.5,10.9)$ & 6.8 & $(5.2,9.5)$ \\
\hline & SFt & 11.2 & $(8.3,13.8)$ & & 8.8 & $(7.6,9.9)$ & 8.5 & $\left(\begin{array}{ll}5.7 & 9.2\end{array}\right)$ \\
\hline & $\mathrm{Ft}$ & 8.1 & $(6.2,12.3)$ & & 9.6 & $(8.6,11.6)$ & 8.0 & $(6.2,11.4)$ \\
\hline \multirow{4}{*}{ FFA $(\mathrm{mmol} / \mathrm{L})$} & GFt & 0.26 & $(0.12,0.33)$ & + & 0.56 & $(0.48,0.76)$ & 0.66 & $(0.55,0.94)^{*}$ \\
\hline & $\mathrm{FFt}$ & 0.23 & $(0.14,0.34)$ & & 0.58 & $(0.37,0.76)$ & 0.62 & $(0.54,0.79)$ \\
\hline & SFt & 0.21 & $(0.16,0.26)$ & & 0.61 & $(0.47,0.72)^{*}$ & 0.81 & $(0.60,0.98)^{*}$ \\
\hline & $\mathrm{Ft}$ & 0.47 & $(0.43,0.83)$ & & 0.71 & $(0.59,0.94)$ & 0.88 & $(0.73,1.00)^{*}$ \\
\hline \multirow{4}{*}{$\mathrm{TG}(\mathrm{mg} / \mathrm{dL})$} & GFt & 55.0 & $(39.5,100.8)$ & & 73.0 & $(60.8,109.3)$ & 54.5 & $(40.3,91.3)$ \\
\hline & $\mathrm{FFt}$ & 57.0 & $(46.8,84.8)$ & & 87.0 & $(63.8,129.8)^{*}$ & 73.5 & $(42.5,100.0)$ \\
\hline & SFt & 78.0 & $(54.0,86.3)$ & & 75.5 & $(60.3,118.0)$ & 64.5 & $(40.3,82.8)$ \\
\hline & $\mathrm{Ft}$ & 76.5 & $(56.8,89.5)$ & & 63.5 & $(52.8,89.0)$ & 42.0 & $(36.0,56.0)$ \\
\hline \multirow{4}{*}{ RLP-TG (mg/dL) } & GFt & 12.2 & $(8.9,18.3)$ & & 21.1 & $(15.7,24.1)$ & 12.0 & $(8.8,18.4)$ \\
\hline & $\mathrm{FFt}$ & 14.9 & $(11.5,22.3)$ & & 24.4 & $(12.2,46.5)^{*}$ & 13.4 & $(9.9,22.3)$ \\
\hline & SFt & 16.6 & $(13.4,22.4)^{*}$ & & 20.6 & $(13.7,31.1)$ & 15.5 & $(9.5,17.9)$ \\
\hline & $\mathrm{Ft}$ & 20.3 & $(16.0,24.8)^{*}$ & & 16.9 & $(12.6,23.5)^{*}$ & 9.7 & $(7.8,10.9)$ \\
\hline \multirow{4}{*}{ RemL-C (mg/dL) } & GFt & 3.10 & $(2.78,8.85)]$ & & 4.80 & $(3.40,8.58)$ & 4.05 & $(3.48,8.23)$ \\
\hline & $\mathrm{FFt}$ & 4.60 & $(3.55,5.60)$ & & 6.80 & $(4.88,8.73)^{*}$ & 6.10 & $(4.93,8.35)^{*}$ \\
\hline & SFt & 5.65 & $(4.18,8.33)$ & {[]$^{\dagger}$} & 6.75 & $(4.83,9.20)$ & 6.35 & $(4.48,9.05)$ \\
\hline & $\mathrm{Ft}$ & 6.35 & ] & & 6.50 & $(4.30,8.90)$ & 4.75 & $(3.98,7.30)$ \\
\hline \multirow{4}{*}{ hTRL - TG (mg/dL) } & GFt & 44.5 & $(28.8,76.0)$ & & 56.5 & $(37.5,90.8)$ & 43.5 & $(24.8,76.8)$ \\
\hline & $\mathrm{FFt}$ & 47.0 & $(33.5,59.8)$ & & 71.0 & $(48.0,93.8)^{*}$ & 60.0 & $(29.3,77.0)$ \\
\hline & SFt & 60.5 & $(44.8,67.8)$ & & 57.5 & $(45.0,89.0)$ & 48.0 & $(29.3,68.8)$ \\
\hline & $\mathrm{Ft}$ & 57.5 & $(41.0,77.8)$ & & 49.5 & $(38.0,76.3)$ & 29.5 & $(24.8,47.0)$ \\
\hline \multirow{4}{*}{ apoB100 (mg/dL) } & GFt & 70.8 & $(60.0,77.6)$ & & 70.9 & $(62.1,78.2)$ & 72.4 & $(63.7,80.2)$ \\
\hline & $\mathrm{FFt}$ & 69.7 & $(54.8,78.5)$ & & 70.6 & $(56.6,83.7)$ & 73.1 & $(57.4,84.8)$ \\
\hline & SFt & 68.6 & $(57.3,82.2)$ & & 70.7 & $(59.7,85.3)$ & 71.8 & $(60.0,87.0)$ \\
\hline & $\mathrm{Ft}$ & 67.0 & $(52.7,77.8)$ & & 69.1 & $(53.4,77.4)$ & 69.5 & $(53.2,78.4)$ \\
\hline \multirow{4}{*}{ apoB48 $(\mu \mathrm{g} / \mathrm{mL})$} & GFt & 2.55 & $(2.18,3.53)^{*}$ & & 2.20 & $(1.75,2.90)$ & 2.40 & $(1.55,3.30)$ \\
\hline & $\mathrm{FFt}$ & 2.95 & $(2.70,4.40)^{*}$ & & 4.50 & $(3.80,5.03)^{*}$ & 3.85 & $(1.75,4.93)^{*}$ \\
\hline & SFt & 6.30 & $(3.05,7.90)^{*}$ & & 4.45 & $(2.65,6.83)^{*}$ & 3.75 & $(1.93,6.00)$ \\
\hline & $\mathrm{Ft}$ & 4.70 & $(4.15,6.30)$ & & 4.25 & $(2.78,6.48)$ & 3.55 & $(2.78,4.85)$ \\
\hline
\end{tabular}

All values are presented as median (Q1, Q3). ${ }^{*} \mathrm{p}<0.05$ compared to the fasting values. ${ }^{\dagger} \mathrm{p}<0.05$ compared between the trials.

Table 4. $\triangle A U C$ for glucose, fructose, insulin, lactate, FFA, TG, RLP-TG, RemL-C, hTRL-TG, apoB100, and apoB48 in Exp 2.

\begin{tabular}{|c|c|c|c|c|c|c|c|c|}
\hline Trial & & GFt & & FFt & & SFt & & Ft \\
\hline \multirow[t]{2}{*}{ Glucose (mg.h/dL) } & 26.5 & $(-27.42,60.84)$ & -0.47 & $(-30.77,5.27)$ & 17.17 & $(-19.31,26.63)$ & -3.92 & $(-17.90,18.82)$ \\
\hline & & \pm & & + & & + & & \\
\hline \multirow[t]{2}{*}{ Fructose (mg.h/dL) } & $-0,55$ & $(-2.27,1.34)$ & 9.83 & $(7.99,11.22)$ & 3.72 & $(1.51,4.55)$ & -0.61 & $(-1.79,0.53)$ \\
\hline & & + & $\sqcup$ & & $\dagger$ & & 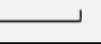 & \\
\hline \multirow{3}{*}{ Insulin $(\mu \mathrm{U} \cdot \mathrm{h} / \mathrm{mL})$} & & \pm & & \pm & & $t$ & 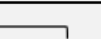 & \\
\hline & 31.1 & $(19.76,43.25)$ & 8.94 & $(5.34,12.76)$ & 25.28 & $(20.66,34.36)$ & -4.55 & $(-15.04,4.60)$ \\
\hline & & $\dagger$ & $\sqcup$ & & \pm & & & \\
\hline L actate $(\mathrm{mg} \cdot \mathrm{h} / \mathrm{dL})$ & -6.65 & $(-14.70,1.55)$ & -1.70 & $(-5.53,22.16)$ & 4.63 & $\left(\begin{array}{ll}-5.39 & 11.74\end{array}\right)$ & -15.00 & $(-29.66,-1.19)$ \\
\hline
\end{tabular}




\begin{tabular}{|c|c|c|c|c|c|c|c|c|}
\hline $\mathrm{FFA}(\mathrm{mmol} \cdot \mathrm{h} / \mathrm{L})$ & -0.16 & $\left(\begin{array}{ll}-0.80 & 0.70\end{array}\right)$ & -0.45 & 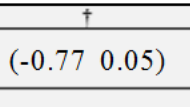 & 0.44 & $\left(\begin{array}{ll}-0.11 & 0.75\end{array}\right)$ & 0.70 & $\left(\begin{array}{lll}0.29 & 1.10\end{array}\right)$ \\
\hline $\mathrm{TG}(\mathrm{mg} \cdot \mathrm{h} / \mathrm{dL})$ & 44.8 & $\frac{\dagger}{(-11.6,74.4)}$ & 101.5 & $(63.5,208.8)$ & 78.3 & $(48.1,136.0)$ & 31.0 & $(4.0,66.5)$ \\
\hline RLP-TG (mg.h/dL) & 37.6 & $(19.1,56.4)$ & 52.2 & $(10.6,94.0)$ & 44.8 & $(31.4,71.0)$ & 28.8 & $(15.1,48.6)$ \\
\hline RemL-C (mg.h/dL) & 2.6 & $(-1.0,6.5)$ & 6.5 & $(4.1,15.5)$ & 7.6 & $(1.8,12.5)$ & 2.7 & $(0.6,5.3)$ \\
\hline hTRL -TG (mg.h/dL) & 16.3 & $\frac{t}{(-35.8,41.4)}$ & $\frac{83.0}{\dagger}$ & $(47.8,154.8)$ & $\frac{t}{53.3}$ & $(20.9,91.6)$ & 9.8 & $(-7.3,39.0)$ \\
\hline apoB100 (mg.h/dL) & -1.5 & $(-4.0,1.9)$ & -0.5 & $(-7.3,4.9)$ & -2.4 & $(-11.1,0.4)$ & -3.4 & $(-9.4,0.6)$ \\
\hline apoB48 $(\mu \mathrm{g} \cdot \mathrm{h} / \mathrm{mL})$ & 1.70 & $(1.41,2.00)$ & $\frac{1.18}{\dagger}$ & $\frac{\dagger}{(0.59,1.63)}$ & 3.70 & $(1.88,4.13)$ & 2.28 & $(1.44,2.85)$ \\
\hline
\end{tabular}

All values are presented as median (Q1, Q3). ${ }^{\dagger} p<0.05$ compared between the trials.

\section{Discussion}

In Exp 1, fructose, when ingested singularly (without fat), ameliorated glycemic and insulinemic response compared with glucose or sucrose. However, fructose had a non-significant effect toward the increase in TG-rich lipoprotein (measured as RemL-C and hTRL-TG) at the end of the experiment. More studies are warranted to elucidate such subtle effects of fructose. In Exp 2, when ingested with fat, fructose again ameliorated glycemic and insulinemic response compared with glucose or sucrose. However, fructose exacerbated and delayed the peak of endogenous TG-rich lipoprotein (hTRL-TG). Although largely non-significant, sucrose was intermediate between fructose and glucose. These results suggest that fructose is favorable for glycemic response, sparing insulin. However, when ingested with fat, fructose may delay and exacerbate postprandial lipidemia and the effect of sucrose may be intermediate between fructose and glucose.

Postprandial lipoprotein metabolism is highly complicated and difficult to analyze separately. However, to analyze postprandial lipidemia effectively, clarifying and discriminating endogenous and exogenous lipoprotein metabolism separately is important. Therefore, we used various indicators of endogenous and exogenous lipoprotein metabolism, such as TG, RLP-TG, RemL-C, hTRL-TG, apoB100, and apoB48. Moreover, apoB48 is a direct index of exogenous lipoprotein number, and apoB100 is an indicator of endogenous lipoprotein number. RLP-TG may be more sensitive for exogenous remnants, whereas RemL-C may be suitable for endogenous remnants [17].

In Exp 1, ingestion of fructose slightly increased the serum concentrations of TG, RemL-C, and hTRL-TG, but not of RLP-TG and apoB48. This suggests that fructose may stimulate the production and/or secretion of endogenous TRL, but not of exogenous TRL. Fructose has been reported to be a better substrate than glucose for fatty acid synthesis in the liver, largely because of its rapid metabolism, which allows fructose to provide more acetyl CoA for fatty acid synthesis than glucose [18]. Ingestion of sucrose (or coingestion of glucose and fructose) had a similar but largely insignificant tendency. Meanwhile, ingestion of glucose did not increase exogenous and endogenous TRL concentration, but non-significantly increased apoB48 concentration at $0.5 \mathrm{~h}$, suggesting transient stimulation of the release of apoB48-containing (probably TG-poor) particles, consistent with our previous results [11]. Fructose absorption is reportedly facilitated by concomitant glucose intake; for example, fructose may be better absorbed from sucrose than from fructose itself [19]. However, in the present study, the serum concentration of fructose did not differ between the $\mathrm{S}$ and GF trials.

In Exp 2, coingestion of sucrose or fructose and fat delayed postprandial lipidemia compared with ingestion of fat alone, but coingestion of glucose and fat did not significantly delay postprandial lipid metabolism. The apoB48 level did not return to baseline even at the end of the experiment $(6 \mathrm{~h})$ after coingestion of fructose and fat. Glucose reportedly stimulated TRL-apoB48 production, with a concomitant moderate increase in fractional clearance when coinfused with Intralipid $^{\mathrm{TM}}$ into the duodenum; this resulted in a net elevation of TRL-apoB48 concentration. However, the TRL-apoB100 concentration, fractional clearance, and production were not significantly affected by glucose. When glucose was replaced by fructose, both TRL-apoB100 and apoB48 production, but not their fractional clearance, were enhanced compared with that observed with Intralipid ${ }^{\mathrm{TM}}$ alone [20]. The impaired clearance of TRL after ingestion of fructose or sucrose is probably, at least partially, mediated by the inhibition of TG 
lipolysis by lipoprotein lipase (LPL). This may also be responsible for the accumulation of cholesterol in the TRL, namely in RemL-C when fat is coingested with sucrose or fructose. Fructose (as monosaccharide or as a component of sucrose) ingested with fat may enhance hepatic TRL production/secretion and delay intestinal TRL production/secretion. Fructose is rapidly fluxed to the liver and is converted to TG; consequently, large VLDL may be overproduced/secreted. Then, at the late postprandial phase, delayed intestinal TRL may compete with overproduced hepatic TRL for the same lipolytic pathway by LPL.

It has been hypothesized that females are protected against fructose-induced hypertriglyceridemia because of a lower stimulation of de novo lipogenesis and lower suppression of lipid oxidation compared with those in men [21]. In healthy nonobese women, the secretion rate of VLDL-TG is higher, but VLDL-apoB100 concentration (i.e., VLDL particle number) is lower compared with men. And the latter offsets increased hepatic VLDL-TG secretion. This indicates that the liver in women secretes fewer but TG-richer VLDL particles than the liver in men [22]. Because TG removal from large, TG-rich VLDL particles via LPL is more efficient than TG removal from small TG-poor particles [23, 24] and increased plasma clearance of VLDL-TG may be responsible for lower VLDL-TG concentration, decreased VLDL-apB100 secretion rate, combined with shorter VLDL-apoB100 residence time in plasma, may be responsible for lower VLDL-apoB100 concentration in women than in men. Thus, in the present study, low to moderate amount of fructose, and to a lesser extent, sucrose delayed postprandial lipoprotein metabolism of ingested fat in young healthy women.

\section{Conclusion}

Ingestion of fructose may increase endogenous TRL-TG concentration, but the effect of sucrose was weaker. Coingestion of fructose and fat delayed postprandial lipidemia compared with ingestion of fat alone. The extent of postprandial lipid response by sucrose coingestion with fat was intermediate between fructose and glucose. Altogether, the present results indicate, when using sugars, such as fructose, glucose, and sucrose, it should be kept in mind that these sugars may have differential effects on carbohydrate and lipoprotein metabolism even in young Japanese women. However, owing to the relatively small number of subjects examined, the present results should be interpreted with caution.

\section{Acknowledgements}

This study was supported by a Grant-in-Aid for Scientific Research (Basic Research C, JSPS KAKENHI Grant Number 24500874) from the Ministry of Education, Culture, Sports, Science and Technology of Japan. We would like to thank Ryuji Hayashi for his technical assistance. We also thank Kotoko Ishiguro, Nami Ito, Kasumi Ohashi, Yuri Imura, Mayuna Suga, and Natsuki Tanabe for their technical cooperation.

\section{References}

[1] Carlson LA, Ericsson M. 1975. Quantitative and qualitative serum lipoprotein analysis. Part 1 . Studies in healthy men and women. Atherosclerosis 21: 417-433.

[2] Asia Pacific Cohort Studies Collaboration. 2004. Serum triglycerides as a risk factor for cardiovascular diseases in the Asia-Pacific region. Circulation 110: 2678-2686.

[3] Bansal S, Buring JE, Rifai N, Mora S, Sacks FM, Ridker PM. 2007. Fasting compared with nonfasting triglycerides and risk of cardiovascular events in women. JAMA 298: 309-316.

[4] Nordestgaard BG, Benn M, Schnohr P, Tybjaerg-Hansen A. 2007. Nonfasting triglycerides and risk of myocardial infarction, ischemic heart disease, and death in men and women. JAMA 298: 299-308.

[5] Nordestgaard BG, Varbo A. 2014. Triglycerides and cardiovascular disease. Lancet 384: 626-35.

[6] Varbo A, Benn M, Tybjaerg-Hansen A, Jorgensen AB, Frikke-Schmidt R, Nordestgaard BG. 2013. Remnant cholesterol as a causal risk factor for ischemic heart disease. $\mathrm{J}$ Am Coll Cardiol 61:427-36.

[7] Welsh JA, Sharma A, Cunningham SA, Vos MB. 2011. Consumption of added sugars and indicators of cardiovascular disease risk among US adolescents. Circulation 123: 249-257.

[8] Lee AK, Binongo JN, Chowdhury R, Stein AD, Gazmararian JA, Vos MB, Welsh JA. 2014. Consumption of less than $10 \%$ of total energy from added sugars is associated with increasing HDL in females during adolescence: a longitudinal analysis. J Am Heart Assoc 3: e000615.

[9] Welsh JA, Sharma A, Abramson JL, Vaccarino V, Gillespie C, Vos MB. 2010. Caloric sweetener consumption and dyslipidemia among US adults. JAMA 303: 1490-1497.

[10] Park YK, Yetley EA. 1993. Intakes and food sources of fructose in the United States. Am J Clin Nutr 58: 737S-747S.

[11] Saito H, Kagaya M, Suzuki M, Yoshida A, Naito M. 2013. Simultaneous ingestion of fructose and fat exacerbates postprandial exogenous lipidemia in young healthy Japanese women. J Atheroscler Thromb 20: 591-600.

[12] Saito H, Kato M, Yoshida A, Naito M. 2015. The ingestion of a fructose-containing beverage combined with fat cream exacerbates postprandial lipidemia in young healthy women. $\mathrm{J}$ Atheroscler Thromb 22: 85-94.

[13] Nabeno Y, Fukuchi Y, Matsutani Y, Naito M. 2007. Influence of aging and menopause on postprandial lipoprotein responses in healthy adult women. J Atheroscler Thromb 14: 142-150.

[14] Matthews DR, Hosker JP, Rudenski AS, Naylor BA, Treacher DF, Turner RC. 1985. Homeostasis model assessment: insulin resistance and beta-cell function from fasting plasma glucose and insulin concentrations in man. Diabetologia 28: 412-419.

[15] Okada M, Saito T, Yoshimura H, Noguchi Y, Ito T, Sasaki H, Hama H. 2005. Surfactant-based homogeneous assay for the measurement of triglyceride concentrations in VLDL and intermediate-density lipoprotein. Clin Chem 51: 1804-1810. 
[16] Nakatani K, Sugimoto T, Masuda D, Okano R, Oya T, Monden Y, Yamashita T, Kawase R, Nakaoka H, Inagaki M, Yuasa-Kawase M, Tsubakio-Yamamoto K, Ohama T, Nishida M, Ishigami M, Komuro I, Yamashita S. 2011. Serum apolipoprotein B-48 levels are correlated with carotid intima-media thickness in subjects with normal serum triglyceride levels. Atherosclerosis 218:226-232.

[17] Sato I, Ishikawa Y, Ishimoto A, Katsura S, Toyokawa A, Hayashi F, Kawano S, Fujioka Y, Yamashita S, Kumagai S. 2009. Significance of measuring serum concentrations of remnant lipoproteins and apolipoprotein B-48 in fasting period. J Atheroscler Thromb 16: 12-20.

[18] Frayn KN, Kingman SM. 1995. Dietary sugars and lipid metabolism in humans. Am J Clin Nutr 62: 250S-261S.

[19] Riby JE, Fujisawa T, Kretchmer N. 1993. Fructose absorption. Am J Clin Nutr 58: 748S-753S.

[20] Xiao C, Dash S, Morgantini C, Lewis GF. 2013. Novel role of enteral monosaccharides in intestinal lipoprotein production in healthy humans. Arterioscler Thromb Vasc Biol 33: 1056-1062.

[21] Tran C, Jacot-Descombes D, Lecoultre V, Fielding BA, Carrel G, Lê KA, Schneiter P, Bortolotti M, Frayn KN, Tappy L. 2010. Sex differences in lipid and glucose kinetics after ingestion of an acute oral fructose load. Br J Nutr 104: 1139-1147.

[22] Magkos F, Patterson BW, Mohammed BS, Klein S, Mittendorfer B. 2007. Women produce fewer but triglyceride-richer very low-density lipoproteins than men. J Clin Endocrinol Metab. 92: 1311-1318.

[23] Fisher RM, Coppack SW, Humphreys SM, Gibbons GF, Frayn KN. 1995. Human triacylglycerol-rich lipoprotein subfractions as substrates for lipoprotein lipase. Clin Chim Acta 236: 7-17.

[24] Streja D, Kallai MA, Steiner G. 1977. The metabolic heterogeneity of human very low density lipoprotein triglyceride. Metabolism 26: 1333-1344. 